\title{
Clinical efficacy of 5-aminolevulinic acid photodynamic therapy in the treatment of moderate to severe facial acne vulgaris
}

\author{
XIANGQI CHEN ${ }^{1,2}$, HONGTAO SONG ${ }^{2}$, SHENGPING CHEN ${ }^{1}$, JING ZHANG ${ }^{2}$, \\ GAOXIANG NIU ${ }^{1}$ and XIANGNONG LIU ${ }^{1}$ \\ Departments of ${ }^{1}$ Dermatology and ${ }^{2}$ Pharmacy, Fuzhou General Hospital, \\ Fuzhou Medical College, Second Military Medical University, Fuzhou, Fujian 350025, P.R. China
}

Received August 1, 2014; Accepted April 22, 2015

DOI: $10.3892 /$ etm.2015.2638

\begin{abstract}
Acne vulgaris is considered as a therapeutic challenge in terms of managing ongoing symptoms and preventing scar formation. Although there are many available treatments for alleviating acne, therapies for resistant or moderate-to-severe forms have been limited to systemic agents that are accompanied by potentially severe side-effects. While, aminolevulinic acid (ALA) photodynamic therapy (PDT) has increasingly been used as a simple and safe therapeutic option of acne vulgaris, the clinical efficacy requires confirmation in further studies. The aim of this study was to investigate the efficacy and safety of 5-ALA-PDT in the treatment of moderate-to-severe facial acne vulgaris. A total of 50 patients with moderate-to-severe facial acne were enrolled in the study and randomly divided equally into a therapy group and a control group. In the therapy group, the patients were treated with 5\% 5-ALA for $1.5 \mathrm{~h}$, followed by three 20 -min doses of infrared radiation once a week; in the control group, the patients were treated with three 20 min doses of infrared radiation without 5-ALA once a week. Both treatments lasted for 3 weeks. The clinical efficacy was determined by evaluating acne lesion counts at weeks 0, 2, 4 and 6 . Total efficacy rate (TER) was the primary endpoint of the study, and was defined as the proportion of the patients whose treatment effectiveness evaluation was cured ( $\geq 90 \%$ of skin lesions improved) and excellent (60-89\% improvement). Adverse effects were recorded throughout the study. The study was completed by 24 patients in the therapy group and 23 patients in the control group. The numbers of acne lesions significantly decreased. The TER of the therapy group was significantly higher than that of the control group at weeks 4 and 6 . Adverse effects were observed in 12 patients
\end{abstract}

Correspondence to: Dr Hongtao Song, Department of Pharmacy, Fuzhou General Hospital, Fuzhou Medical College, Second Military Medical University, 156 Xierhuanbei Road, Fuzhou, Fujian 350025, P.R. China

E-mail: shengpingchencn@163.com

Key words: facial acne, photodynamic therapy, infrared radiation, 5-aminolevulinic acid of the therapy group and 2 patients of the control group. In the therapy group the most common adverse effect was a burning sensation $(n=7)$, followed by transient hyperpigmentation $(n=3)$ and acute acneform lesions $(n=2)$, while in control group, the 2 patients experienced flushing and dryness. The adverse effects were all cured by a symptomatic approach prior to the end of the study. 5-ALA-PDT combined with infrared radiation is an effective and safe therapy for moderate-to-severe facial acne.

\section{Introduction}

Acne is a human chronic inflammatory skin disease that typical presents with seborrhea, comedones, papules, pustules and nodules. Its prevalence ranges from 70 to $87 \%$ (1). Furthermore, acne can lead to psychological and social problems, and seriously affect a person's quality of life. Therefore, researchers are focused on finding safe and effective methods for treating acne vulgaris.

Numerous different treatments are available for acne, including benzoyl peroxide, antibiotics, oral or topical retinoids, antiseborrheic medications and hormonal treatments. However, these drugs have limitations in practice, for example, retinoids have certain side-effects, and antibiotics may lead to resistance and affect female fertility (2). Therefore, light therapy, a therapeutic method that includes visible light therapy, photodynamic therapy (PDT) and laser therapy, has widespread clinical value in the treatment of acne $(3,4)$.

Propionibacterium acnes, an important conditioned pathogen, is the target of the different kinds of acne phototherapies. $P$. acnes synthesizes and stores porphyrins, including protoporphyrin and coproporphyrin. Porphyrins are endogenous photosensitive materials that absorb light energy within a specific wavelength range; however the amount of porphyrins produced by $P$. acnes is relatively low (5). Therefore, the topical application of exogenous materials such as the porphyrin precursor 5-aminolevulinic acid (ALA) to the skin lesions is necessary. ALA is absorbed and metabolized into protoporphyrin IX, which accumulates in sebaceous glands and epithelial cells. Porphyrins transfer energy to oxygen, to generate active oxygen molecules, such as singlet oxygen and superoxide anion free radicals, which are electrophilic and effectively oxidize molecules to cause toxicity in epithelial 
cells $(6,7)$. Studies on the optimal drug concentration, exposure dose, and contact time for East Asian populations are in the exploratory stage. The present study investigated the use of 5-ALA-PDT to treat 24 patients with acne vulgaris from June 2011 to December 2012.

\section{Materials and methods}

Subjects. A total of 50 patients were randomly divided equally into a control group and a therapy group. This study was conducted in accordance with the Declaration of Helsinki and with approval from the Ethics Committee of Fuzhou General Hospital (Fuzhou, China). Written informed consent was obtained from all participants.

Enrollment criteria. The acne vulgaris of the patients was graded in terms of property and severity as follows: Low grade, which presented with only acne; moderate grade, which was acne that presented with inflammatory papules and pustules; and severe grade, which is acne that presented with inflammatory papules, nodules, cysts and scars.

Exclusion criteria. The exclusion criteria were as follows: i) use of any topical antibiotics within 2 weeks of the study or intake of systemic oral antibiotics within 4 weeks of the study; ii) use of systemic retinoids within 6 months of the study; iii) porphyria or facial atopic dermatitis; iv) pregnancy or lactation; v) history of keloid or photosensitivity disorders; vi) photosensitive eczema or autoimmune diseases; and vii) use of anti-acne medication such as prophylactics, glucocorticoid and photosensitizers.

Treatment methods. The 5\% ALA solution was prepared by Shanghai Fudan-Zhangjiang Bio-Pharmaceutical Co., Ltd. (Shanghai, China). Prior to ALA application, the skin was cleansed with $70 \%$ isopropyl alcohol. Then, $20 \%$ topical ALA was applied for 90 min under plastic film occlusion and exposed three times for $20 \mathrm{~min}$ to red light (LED-IB photodynamic therapy instrument, Wuhan Yage Optic and Electronic Technique Co. Ltd., Wuhan, China; wavelength, $633 \pm 10 \mathrm{~nm}$; energy density, $10 \mathrm{~mW} / \mathrm{cm}^{2}$; standard of energy, $120 \mathrm{~J} / \mathrm{cm}^{2}$ ) once a week. The control group was exposed to red light three times for 20 min once a week. Both treatments were for 3 weeks.

Side-effects, including itching, pain, erythema, hyperpigmentation and exfoliation, were recorded during the course of treatment. Clinical photographs were taken prior to and following treatment and at every follow-up every 2 weeks for 6 weeks.

Clinical evaluation. The acne of each patient was evaluated using an inflammatory acne score modified from previously described criteria (8). The classifications used in this study accounted for both the number and the size of the lesions. The number of comedones, inflammatory comedones, papules, pustules, nodules and cysts in each test area were recorded. The effects were evaluated in terms of the reduction rate of the acne lesions. Reduction rate was calculated as follows: Reduction rate $(\%)=$ (numbers of comedones before treatment - numbers of comedones after treatment)/number of comedones before treatment $x$ 100. Skin lesions with $\geq 90 \%$ improvement were classified as cured, skin lesions with $60-89 \%$ improvement were classified as excellent effect, skin lesions with $30-59 \%$ improvement were classified as fair effect and skin lesions with $<30 \%$ improvement or exacerbations were classified as no effect. The total effective rate (TER) was computed as follows: TER $(\%)=$ (number of cured cases + excellent effect cases)/total number of cases $\mathrm{x} 100$.

Statistical analysis. The data were analyzed using SPSS software version 13.0 (SPSS, Inc., Chicago, IL, USA) and a $\chi^{2}$ test was used for two-sample comparisons. A rank sum test was used to compare ages and courses among multiple groups. Differences with $\mathrm{P}<0.05$ were considered statistically significant.

\section{Results}

General data. Of the 25 patients in the therapy group, 24 patients (13 males and 11 females) completed the course and 1 dropped out of the study for an undisclosed reason. The age of the patients who completed the ALA-PDT treatment ranged from 18 to 33 years, with a mean age of 23.57 years. The courses ranged from 2 months to 11 years, with a mean course duration of 13.25 months. Of the 25 patients in the control group, 23 cases (12 males and 11 females) completed the course. Two cases dropped out of the treatment because of side-effects and/or poor effect. The age of the patients who completed the control treatment ranged from 19 to 32 years, with a mean age of 24.12 years. The courses ranged from 1 month to 12 years, with a mean of 12.36 months. The two groups did not significantly differ in terms of age distribution, gender and courses $(\mathrm{P}>0.05)$.

Clinical effects. The effects of treatment in two patients from the therapy group are shown in Figs. 1 and 2. In the therapy group, TERs at 2, 4 and 6 weeks were 54.2, 75.0 and 83.3\%, respectively, whereas those in the control group were 26.1, 43.5 and $56.5 \%$, respectively. When the TERs of the treatment and control group were compared, the $\chi^{2}$ values were $3.845,4.846$ and 4.037 and the P-values were $0.050,0.028$ and 0.045 , respectively. This result indicates that the 4- and 6-week efficacy rates significantly differed between the two groups, and were greater in the therapy group than in the control group (Table I).

Adverse effects. In the ALA-PDT group, 7 patients experienced burning sensations, pain, erythema and edema with 1-4 days of red light therapy. The side-effects were treated with saline compresses. Three patients had transient hyperpigmentation and were advised to avoid light. Two patients developed small pimples and were diagnosed with acute acneform lesions, which were topically treated successfully with mupirocin ointment. These two patients completed the course and were cured without any adverse effects. In the control group, two patients experienced flushing and dryness of the face. Following treatment with a saline compress, the evident hyperpigmentation and scars disappeared.

\section{Discussion}

Light therapies such as PDT have played an important role in dermatological practice. These procedures offer alternatives to 
Table I. Comparison of clinical effects in patients with moderate or severe acne vulgaris at 2, 4 and 6 weeks in the two groups.

\begin{tabular}{llcccccccr}
\hline Weeks & Groups & Number & Cured & $\begin{array}{c}\text { Excellent } \\
\text { effect }\end{array}$ & $\begin{array}{c}\text { Fair } \\
\text { effect }\end{array}$ & $\begin{array}{c}\text { No } \\
\text { effect }\end{array}$ & $\begin{array}{c}\text { Total effective } \\
\text { rate }(\%)\end{array}$ & $\chi^{2}$ value & P-value \\
\hline 2 & Treatment & 24 & 3 & 10 & 9 & 2 & 54.2 & 3.845 & 0.050 \\
& Control & 23 & 1 & 5 & 13 & 4 & 26.1 & & \\
4 & Treatment & 24 & 11 & 7 & 4 & 2 & 75.0 & 4.846 & 0.028 \\
& Control & 23 & 4 & 6 & 10 & 3 & 43.5 & & \\
6 & Treatment & 24 & 15 & 5 & 3 & 1 & 83.3 & 4.037 & 0.045 \\
& Control & 23 & 6 & 7 & 8 & 2 & 56.5 & & \\
\hline
\end{tabular}
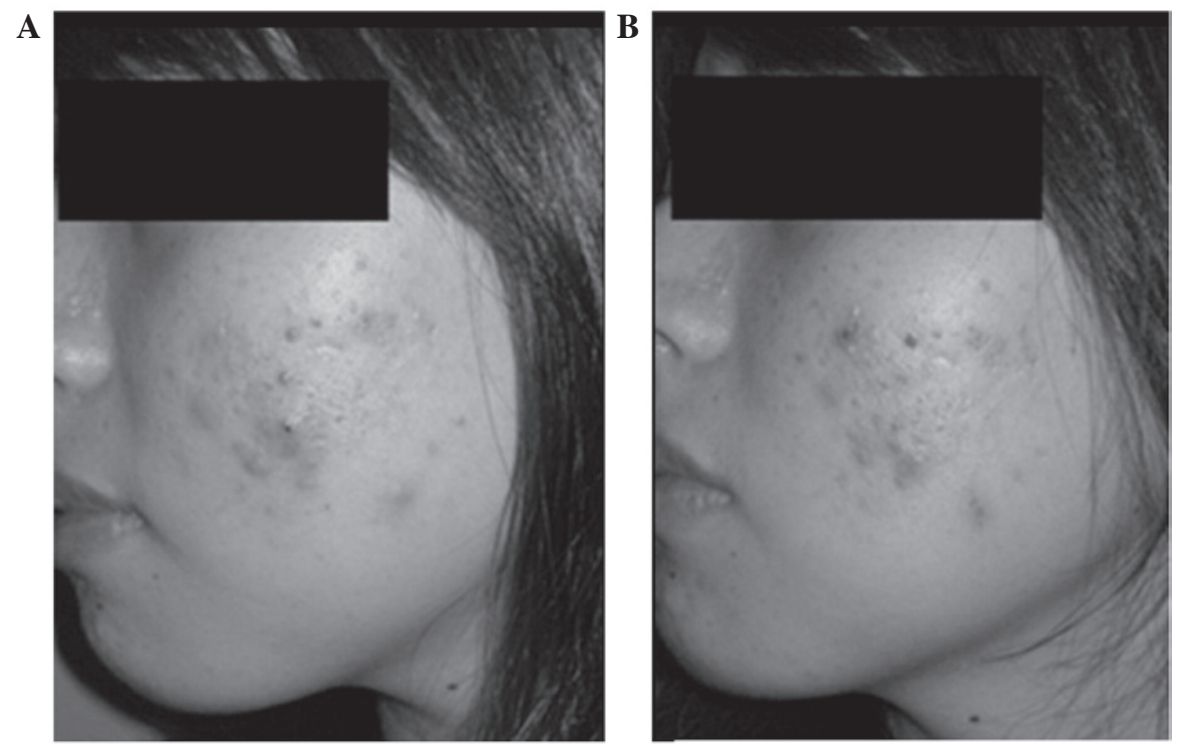

Figure 1. Effect in a female patient. (A) The left side of the face presented red papula and had four pimples prior to treatment with ALA-PDT. (B) The red papula darkened and pimples faded after three treatments. ALA, aminolevulinic acid; PDT, photodynamic therapy.
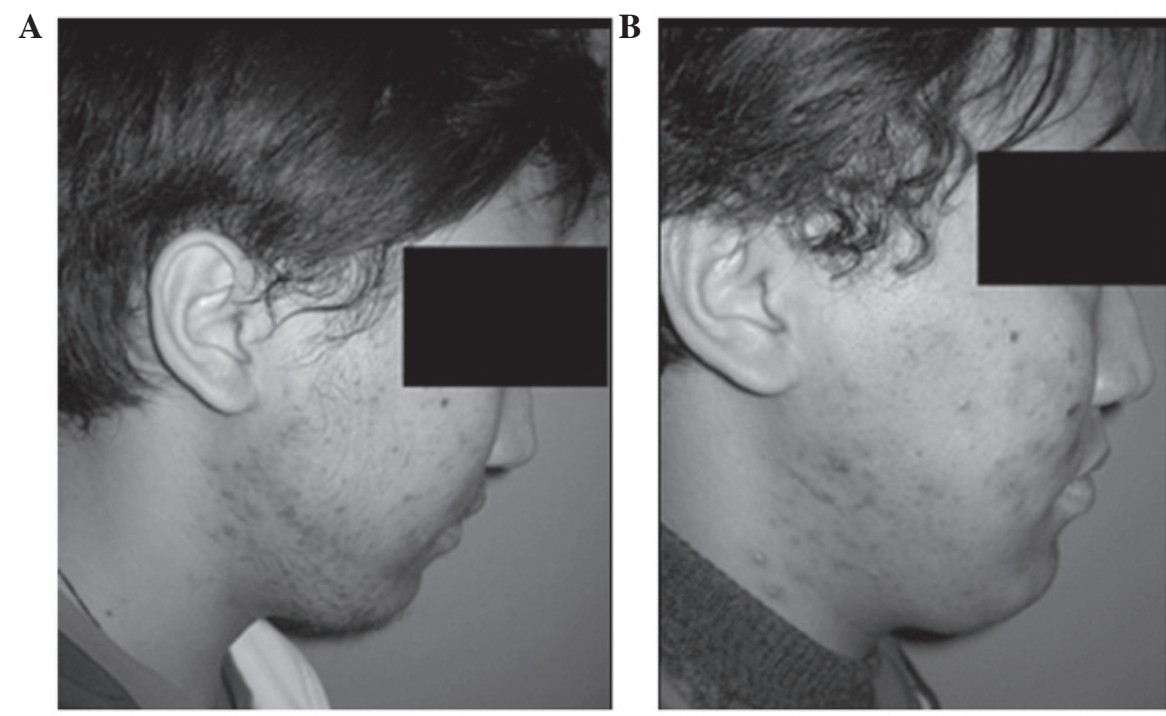

Figure 2. Effect in a male patient. (A) The right side of the face had dull red papula and 12 red/pink nodules prior to treatment with ALA-PDT. (B) The dull red papula and nodules had faded away, and only brown-colored traces remained after three treatments. ALA, aminolevulinic acid; PDT, photodynamic therapy.

people who seek topical treatments, a quicker onset of action, non-serious side-effects and decreased antibiotic resistance rates. Following 5-ALA-PDT, 5-ALA is absorbed by epithelial cells and synthesized into protoporphyrin IX through the 
hemachrome biosynthetic pathway. Photoactivated porphyrin is produced from the formation of singlet oxygen and other potent oxidizers that induce transient antimicrobial and anti-inflammatory effects $(9,10)$. However, this also inhibits secretion by the sebaceous gland and damages its structure (11-13). In a review of 16 randomized and 3 controlled trials, Haedersdal et al (14) investigated the use of physical therapy such as PDT, infrared lasers and intense pulsed light, in the treatment of 587 patients with acne vulgaris. The results indicated that optical treatments for acne vulgaris have short-term efficacy, with the most consistent outcomes achieved through PDT, which minimizes scar formation. The majority of the patients developed ALA photosensitivity that resolved within $24 \mathrm{~h}$ (14). In addition, Shaaban et al (15) observed 30 patients with nodulocystic acne vulgaris and compared the effects of intralesional ALA in combination with intense pulsed light (IPL) with the effects of IPL alone. The results demonstrated that ALA-PDT treatment significantly reduces acne lesions to a greater extent than the IPL treatment alone; thus, the authors concluded that PDT is effective in treating acne vulgaris when combined with ALA (15).

The clinical effectiveness of treating acne vulgaris with 5-ALA-PDT is dependent upon the ALA concentration, contact time, light source and dose. Hongcharu et al performed an open-labeled prospective study involving 22 patients with acne vulgaris treated with ALA and red light (16). The patients were treated with $20 \%$ topical ALA with 3 h of occlusion with a plastic film, and $150 \mathrm{~J} / \mathrm{cm}^{2}$ of broadband light (550-700 nm). The results indicated significant clearance of the acne at 20 weeks after four treatments and at 10 weeks after a single PDT treatment using ALA with red light. The results of a study conducted by $\mathrm{Oh}$ et al suggested that the outcome of treatment with $10 \%$ topical 5-ALA is better with $3 \mathrm{~h}$ occlusion than with $0.5 \mathrm{~h}$ occlusion in acne vulgaris (17). A study by Wang et al also supported the use of this regimen for treating common acne vulgaris (18). However, Yin et al suggested that the high cost of this therapy in China would discourage patients from undergoing this treatment (19). In the current study, 5\% 5-ALA with $1.5 \mathrm{~h}$ of occlusion and $20 \mathrm{~min}$ of red light three times once a week was used. The total efficacy rates at 2, 4 and 6 weeks showed a significant difference between the control group and the therapy group. This indicates that ALA-PDT is effective against acne vulgaris.

ALA-PDT is not a minor procedure, and has some side-effects, such as pain, edema and burning and itching sensations, even erythema, hyperpigmentation and exfoliation (20-22), which are of concern to patients. A review found that every patient experienced a burning sensation that lasted for several minutes ( $\sim \mathrm{min})$ during ALA-PDT treatment, however this symptom eventually resolved during the course of the treatment. Additionally, almost each patient suffered from mild or moderate erythema in the affected area that lasted for 1-5 days; however, most of the patients $(85.46 \%)$ recovered within 2 days. Higher ALA concentrations correlate with longer persistence of the erythema (23).

In the present study, 7 cases presented with different degrees of burning sensation, pain, erythema and edema, 3 cases had hyperpigmentation, and 2 cases had acute acne lesions. It is hypothesized that these symptoms, as well as the superficial capillary vasodilatation, were the body's response to the ALA-PDT and red light, which required only minor treatment. The hyperpigmentation may be associated with the ALA concentration or light dose. Photosensitizers accumulate in the epidermis to cause skin exfoliation. Generally, this symptom appeared within 3-4 days after treatment, but resolved within 1 month. In addition, patients were asked to avoid light with $24 \mathrm{~h}$ after treatment. Furthermore, the patients were asked to apply an antioxidant such as vitamin $\mathrm{C}$ prior to going outside to prevent hyperpigmentation.

In conclusion, the results of the present study indicate that 5\% 5-ALA with $1.5 \mathrm{~h}$ of occlusion in addition to irradiation with red light for $20 \mathrm{~min}$ three times once a week is an effective treatment for moderate-to-severe acne vulgaris. Moreover, the few side-effects of 5-ALA-PDT and the short therapy course are likely to improve patient compliance. Additionally, 5\% 5-ALA is easy to apply, the procedure is simple and the treatment does not induce resistance. Thus, 5-ALA-PDT treatment is recommended for clinical application in the treatment of acne vulgaris.

\section{References}

1. Song WF, Liu Z, Lin SJ, et al: Photodynamic therapy in acne with 5-aminolevulinic acid: Analysis of clinical effect. Zhong Guo Ji Guang Yi Xue Za Zhi 19: 223-226, 2010 (In Chinese).

2. Ozolins M, Eady EA, Avery A, Cunliffe WJ, O'Neill C, Simpson NB and Williams HC: Randomized controlled multiple treatment comparison to provide a cost-effectiveness rationale for the selection of antimicrobial therapy in acne. Health Technol Assess 9: iii-212, 2005.

3. Gold MH: Efficacy of lasers and PDT for the treatment of acne vulgaris. Skin Therapy Lett 12: 1-6, 9, 2007.

4. Rai R and Natarajan K: Laser and light based treatments of acne. Indian J Dermatol Venereol Leprol 79: 300-309, 2013.

5. Elman M, Slatkine M and Harth Y: The effective treatment of acne vulgaris by a high-intensity, narrow band 405-420 nm light source. J Cosmet Laser Ther 5. 111-117, 2003.

6. Nestor MS, Gold MH, Kauvar AN, et al: The use of photodynamic therapy in dermatology: Results of a consensus conference. J Drugs Dermatol 5: 140-154, 2006.

7. Klein A, Babilas P, Karrer S, Landthaler M and Szeimies RM: Photodynamic therapy in dermatology - an update 2008. J Dtsch Dermatol Ges 6: 839-846, 2008.

8. Tzung TY, Wu KH and Huang ML: Blue light phototherapy in the treatment of acne. Photodermatol Photoimmunol Photomed 20: 266-269, 2004

9. Lee Y and Baron ED: Photodynamic therapy: Current evidence and applications in dermatology. Semin Cutan Med Surg 30: 199-209, 2011.

10. Harris F and Pierpoint L: Photodynamic therapy based on 5 -aminolevulinic acid and its use as an antimicrobial agent. Med Res Rev 32: 1292-1327, 2012

11. Mariwalla K and Rohrer TE: Use of laser and light-based therapies for treatment of acne vulgaris. Lasers Surg Med 37: 333-342, 2005.

12. Kosaka S, Miyoshi N, Akilov OE, Hasan T and Kawana S: Targeting of sebaceous glands by $\delta$-aminolevulinic acid-based photodynamic therapy: An in vivo study. Lasers Surg Med 43: 376-381, 2011.

13. Kostović K, Pastar Z, Ceović R, Mokos ZB, Buzina DS and Stanimirović A: Photodynamic therapy in dermatology: Current treatments and implications. Coll Antropol 36: 1477-1481, 2012.

14. Haedersdal M, Togsverd-Bo K and Wulf HC: Evidence-based review of lasers, light sources and photodynamic therapy in the treatment of acne vulgaris. J Eur Acad Dermatol Venereol 22: 267-278, 2008.

15. Shaaban D, Abdel-Samad Z and El-Khalawany M: Photodynamic therapy with intralesional 5-aminolevulinic acid and intense pulsed light versus intense pulsed light alone in the treatment of acne vulgaris: A comparative study. Dermatol Ther 25: 86-91. 2012. 
16. Hongcharu W, Taylor CR, Chang Y, Aghassi D, Suthamjariya K and Anderson RR: Topical ALA-photodynamic therapy for the treatment of acne vulgaris. J Invest Dermatol 115: 183-192, 2000.

17. Oh SH, Ryu DJ, Han EC, Lee KH and Lee JH: A comparative study of topical 5-aminolevulinic acid incubation times in photodynamic therapy with intense pulsed light for the treatment of inflammatory acne. Dermatol Surg 35: 1918-1926, 2009.

18. Wang XL, Wang HW, Zhang LL, Guo MX and Huang Z: Topical ALA PDT for the treatment of severe acne vulgaris. Photodiagnosis Photodyn Ther 7: 33-38, 2010.

19. Yin R, Hao F, Deng J, Yang XC and Yan H: Investigation of optimal aminolaevulinic acid concentration applied in topical aminolaevulinic acid-photodynamic therapy for treatment of moderate to severe acne: A pilot study in Chinese subjects. Br J Dermatol 163: 1064-1071, 2010.
20. Serrano G, Lorente M, Reyes M, et al: Photodynamic therapy with low-strength ALA, repeated applications and short contact periods (40-60 min) in acne, photoaging and vitiligo. J Drugs Dermatol 8: 562-568, 2009.

21. Bissonnette R: Treatment of acne with photodynamic therapy. G Ital Dermatol Venereol 146: 445-456, 2011.

22. Mei X, Shi W and Piao Y: Effectiveness of photodynamic therapy with topical 5-aminolevulinic acid and intense pulsed light in Chinese acne vulgaris patients. Photodermatol Photoimmunol Photomed 29: 90-96, 2013.

23. Sakamoto FH, Torezan L and Anderson RR: Photodynamic therapy for acne vulgaris: A critical review from basics to clinical practice: part II. Understanding parameters for acne treatment with photodynamic therapy. J Am Acad Dermatol 63: 195-211, 2010 . 